\title{
INFINITE MATRICES ASSOCIATED WITH DIFFRACTION BY AN APERTURE*
}

\author{
$\mathrm{BY}$ \\ WILHELM MAGNUS \\ New York University
}

1. Introduction and summary. As an example of their "variational method", LEVINE and SCHWINGER [1] investigated a boundary value problem which arises from the diffraction of a plane scalar (acoustical) wave by a plane screen with a circular aperture. It is equivalent to the problem of finding the field of a freely vibrating circular disk. A full discussion of the physical problems was given by Bouwkamp [2]. Let $z$, $\rho, \theta$ be cylindrical coordinates and let $z=0$ be the plane occupied by the screen. Let $z=0,0 \leqq \rho<a$ define the aperture (or the vibrating disk). The diffracted field is given by a function $u$ which satisfies $\nabla^{2} u+k^{2} u=0$ (with a constant $k$ ) everywhere except for $z=0$ and at infinity satisfies a Sommerfeld radiation condition. For $z=0$, $u$ must satisfy the "mixed" boundary conditions $u=0$ for $\rho>a$ and $\partial u_{\prime}^{\prime} \partial z=v_{0}$ with a given constant value $v_{0}$ for $0 \leqq \rho<a$. These conditions determine $u$ uniquely. For $z=0,0 \leqq \rho<a, u=\Phi(\rho)$ becomes a function of $\rho$ only, and if $\Phi(\rho)$ is known or even if only $C_{0} \Phi(\rho)$ with an undetermined constant factor $C_{n}$ is known, $u$ can be determined everywhere; see formulas (A.1), (A.2), (A.3) in [1].

Levine and Schwinger [1] show that the ratio of the energy transmitted through the aperture to the energy incident on the aperture is the imaginary part of the complex transmission coefficient $T^{*}$, which is a quotient of two integrals involving $\Phi(\rho)$ quadratically. As a functional of $\Phi(\rho), T^{*}$ becomes stationary for the correct function $\Phi$ which determines $u$. Levine and Schwinger find approximate values for $T^{*}$ by expanding first $\Phi(\rho)$ in an infinite series of auxiliary functions (see 3.1 and 3.2) with coefficients $D_{m}$. Then $T^{*}$ becomes a linear form in the $D_{m}$ (see 3.10 ), and the unknowns $D_{m}$ are determined by an inhomogeneous system of infinitely many linear equations with a coefficient matrix $L$ (see 3.4, 3.5). In [1], these equations are solved "section wise", using the first $l=1,2,3, \cdots$ equations to determine the first $l$ unknowns. All quantities $D_{m}$, $T^{*}, L$ are power series in $\beta=k a / 2$, and I evine and Schwinger compute the first coefficients of the expansion of $T^{*}$ in a power series in $\beta$ which were determined independently by Bouwkamp [2], who used spheroidal wave functions.

It will be shown that the algebraic properties of the matrix $L$ make it possible not only to find approximate values for $T^{*}$ as in [1] but also to determine $\Phi(\rho)$. This is due to the fact that $L$ factorizes in a product $L^{(0)} S$, where $L^{(0)}$ is the matrix for the static case $k=0$ and where $S$ can be inverted by solving finite recurrence relations. The details are stated in lemma 1 and theorem 1 of section 3. Lemma 2 gives additional algebraic relations. Problems of convergence and uniqueness are settled in section 5 . These depend largely on an investigation of the properties of $L^{(0)}$ which is carried through in section 4. There it is shown that in the limiting cases $k=0$ and $k=\infty$ the matrices $L^{(0 .}$ and $L^{(\infty)}$ of the linear equations also arise from a problem of moments. This also makes it possible to prove that the variational method for the calculation of the transmission

${ }^{*}$ Received March 27, 1952. This work was performed at Washington Square College of Arts and Science, New York University, and was supported in part by Contract No. AF-19(122)-42 with the United States Air Force through sponsorship of Geophysical Research Division, Air Force Cambridge Research Center, Air Materiel Command. 
coefficient will work even for $k=\infty$ where the linear equations for the $D_{m}$ do not have any solution at all.

2. Notations. The elements of (infinite) matrices are denoted by subscripts $n$, $m=0,1,2, \cdots$ where $n$ denotes the rows and $m$ denotes the columns. A vector with components $x_{m}$ is denoted by $\left\{x_{m}\right\}$. We also use the notations

$$
\begin{gathered}
(a)_{n}=\Gamma(a+n) / \Gamma(a)=a(a+1) \cdots(a+n-1) ; \quad(a)_{0}=1, \\
F(a, b ; c ; z)=\sum_{n=0}^{\infty} \frac{(a)_{n}(b)_{n}}{(c)_{n} n !} z^{n},
\end{gathered}
$$

where $\Gamma$ denotes the gamma function and $F$ denotes the hypergeometric series. For results needed here see Whittaker and Watson [3] and Bailey [4].

3. Algebraic properties of the linear equations. Let

$$
\Phi(\rho)=-\frac{1}{2} a C_{0} \sum_{m=0}^{\infty} x_{m}\left(1-\rho^{2} / a^{2}\right)^{m+1 / 2}
$$

be the expansion of the field $\Phi(\rho)$ in the aperture in terms of powers of $1-\rho^{2} / a^{2}$. Here $C_{0}$ denotes an undetermined constant and

$$
-\frac{1}{2} a x_{m}=D_{m}
$$

where the $D_{m}$ are the unknowns used by Levine and Schwinger [1]. The linear equations for the $x_{m}$ as obtained from the variational method can be written as follows:

Let $p, q=0,1,2, \cdots$ and let $L^{(2 p)}, L^{(2 q+3)}$ be infinite matrices with elements $l_{n, m}^{(2 p)}$, $l_{n, m}^{(2 a+3)}$ defined by

$$
\begin{aligned}
l_{n, m}^{(2 p)} & =(-1)^{p} \pi^{1 / 2} A(n, m, p) / B(n, m, p), \\
l_{n, m}^{(2 a+3)} & =i(-1)^{a} \pi^{1 / 2} A(n, m, q+3 / 2) / B(n, m, q+3 / 2),
\end{aligned}
$$

where, for any values of $n, m, t$

$$
\begin{aligned}
& A(n, m, t)=\Gamma(n+3 / 2) \Gamma(m+3 / 2) \Gamma(n+m+2 t+1), \\
& B(n, m, t)=4 \Gamma(t+1) \Gamma(n+t+1) \Gamma(m+t+1) \Gamma(n+m+t+5 / 2) .
\end{aligned}
$$

Let $L$ be the matrix

$$
L=\sum_{p=0}^{\infty} \beta^{2 p} L^{(2 p)}+\sum_{q=0}^{\infty} \beta^{(2 Q+3)} L^{(2 q+3)},
$$

the general element $l_{n, m}=l_{n, m}(\beta)$ of which is a power series in $\beta=\frac{1}{2} k a$. Then

$$
\sum_{m=0}^{\infty} l_{n, m} x_{m}=(n+3 / 2)^{-1} \text {. }
$$

Let $\xi$ denote the vector with the components $x_{m}$ and let $\xi^{(r)}, r=0,1, \cdots$ be the vector with the components $x_{m}^{(r)}$ where

$$
x_{m}=\sum_{r=0}^{\infty} \beta^{r} x_{m}^{(r)} .
$$

Let $\eta^{(0)}$ denote the vector with the components $1 /(m+3 / 2)$. Comparing the coefficients of $\beta^{r}, r=0,1, \cdots$, on both sides of (3.6) we find

$$
L^{(0)} \xi^{(0)}=\eta^{(0)}, \quad L^{(0)} \xi^{(1)}=0 .
$$


and, for $r=2,3,4, \cdots$ :

$$
L^{(0)} \xi^{(r)}+L^{(2)} \xi^{(r-2)}+\cdots+L^{(r)} \xi^{(0)}=0 .
$$

If

$$
T^{*}=\sum_{m=0}^{\infty} x_{m} /(m+3 / 2),
$$

the transmission coefficient $T$ becomes

$$
T=\beta / 2 \operatorname{Im} T^{*}
$$

where Im denotes the imaginary part. We shall now show that $L^{(0)}$ is a common left hand factor of all the matrices $L^{(2 p)}, L^{(2 q+3)}$, such that the right hand factor is a bounded matrix.

Lemma 1. Let $p=1,2,3, \cdots$ and $q=0,1,2, \cdots$, and let $S^{(2 p)}=\left(s_{n, m}^{(2 p)}\right)$ and $S^{(2 a+3)}=\left(s_{n, m}^{(2 a+3)}\right)$ be the matrices defined by

$$
\left.\begin{array}{rll}
s_{n, m}^{(2 p)}=0 & \text { if } & n>p+m \\
s_{n, m}^{(2 q+3)}=0 & \text { if } & n>q
\end{array}\right\}
$$

and otheruise

$$
\begin{aligned}
s_{n, m}^{(2 p)} & =(-1)^{p} G(n, m, p) / H(n, m, p), \\
s_{n, m}^{(2 a+3)} & =i(-1)^{a} G(n, m, q+3 / 2) / H(n, m, q+3 / 2),
\end{aligned}
$$

where, for any values of $n, m, t$

$$
\begin{aligned}
& G(n, m, t)=(-t+3 / 2)_{n} \Gamma(2 t-n+m) \Gamma(m+3 / 2) \\
& H(n, m, t)=\Gamma(t+1) \Gamma(t) \Gamma(t+m-n+1) \Gamma(t+m+3 / 2)(3 / 2)_{n} .
\end{aligned}
$$

Then

$$
L^{(2 p)}=L^{(0)} S^{(2 p)}, \quad L^{(2 q+3)}=L^{(0)} S^{(2 q+3)} .
$$

Proof: The element in the $n$-th row and $m$-th column of $L^{(0)} S^{(2 p)}$ is

$$
\frac{\sqrt{\pi}}{4} \frac{(-1)^{p}}{p !(p-1) !} \frac{\Gamma(n+3 / 2)}{n !} \frac{\Gamma(m+3 / 2)}{\Gamma(m+p+3 / 2)} \sum
$$

where, because of (2.1) and simple properties of the Gamma function

$$
\begin{aligned}
\sum_{n, m} & =\sum_{r=0}^{p+m} \frac{(n+r !)}{r !} \frac{\Gamma(r+3 / 2)}{\Gamma(n+r+5 / 2)} \frac{(-p+3 / 2)_{r}}{(3 / 2)_{r}} \frac{\Gamma(2 p+m-r)}{\Gamma(p+m-r+1)} \\
& =\frac{n ! \Gamma(2 p+m) \Gamma(3 / 2)}{(p+m) ! \Gamma(n+5 / 2)} \sum_{r=0}^{p+m} \frac{(n+1)_{r}}{r !} \frac{(3 / 2-p)_{r}}{(n+5 / 2)_{r}} \frac{(-p-m)_{r}}{(1-m-2 p)_{r}}
\end{aligned}
$$

The sum in (3.18) can be computed by using Saalschuetz's formula (cf. Bailey [4] for a simple proof) which can be written in the form

$$
\begin{gathered}
\sum_{r=0}^{k} \frac{(a)_{r}(b)_{r}(-k)_{r}}{r !(c)_{r}(1+a+b-c-k)_{r}}=\frac{(c-a)_{k}(c-b)_{k}}{(c)_{k}(c-a-b)_{k}} \\
(k=0,1,2, \cdots ; c \neq 0,-1-2, \cdots-k-1 ; 1+a+b-c \neq 1,2, \cdots, k)
\end{gathered}
$$


Taking $a=n+1 . b=-p+32 . c=52+r . ?=p+m,(3.19)$ gives for $\sum_{\ldots . . .}$ in (3.17)

$$
\sum_{n, m}=\frac{n ! \Gamma(2 p+m+\Gamma 3 \cdot 2)}{(p+m) ! \Gamma(n+5 \cdot 2} \frac{3 \cdot 2 !+p+1)_{m-1}}{\left(n-52_{m+p}(p)_{m+p}\right.} .
$$

From (3.20) and (3.16) it follows that $L^{\left(2 D^{\prime}\right.}=L^{0} S^{2 t}$. The proof of $L^{(2 D+3)}=L^{(0)} S^{(2 D+3)}$ follows by the same method.

The elements of the matrices $S^{2 q^{-3}}$ are zero except for those in the first $q$ rows. This is not true for the $S^{(2 p)}$ but the following lemma shows that $S^{\left(2 p^{\prime}\right.}$ is a nolynomial in $S^{(2)}$ apart from right hand factors which are either the identity or of the type of the $S^{(2 q+3)}$.

We have:

Lemma 2. Let $p, t=1,2,3, \cdots$ and let $R^{(t)}$ be the matrix for which the element in the first row and $m$-th column is

$$
\frac{(-1)^{t+1}}{(t-1 / 2) t !(t-1) !} \frac{\Gamma(m+3 / 2) \Gamma(2 t+m+1)}{\Gamma(m+t+3 / 2)(t+m+1) !}
$$

all other elements of $R^{(t)}$ being zero. Then

$$
\begin{gathered}
S^{(2)} S^{(2 t)}-\frac{t+1}{1-2 t} S^{(2 t+2)}=R^{(t)}, \\
S^{(2 t+2)}=\sum_{\mu=0}^{t}(-2)^{\mu+1}\left[(-t+1 / 2)_{\mu+1} /(-1-t)_{\mu+1}\right]\left\{S^{(2)}\right\}^{\mu} R^{(t-\mu)},
\end{gathered}
$$

where, for $\mu=t, R^{(0)}$ denotes $S^{(2)}$. In general,

$$
S^{(2 p)} S^{(2 t)}-\frac{(t+p) !}{p ! t !} \frac{\Gamma(3 / 2) \Gamma(-t-p+3 / 2 !}{\Gamma(-p+3 / 2) \Gamma(-t+3 / 2 !} S^{(2 p+2 t)}
$$

is a matrix in which all elements are zero except those in the first $p$ rows.

The proof of lemma 2 follows again from Saalschuetz's formula. We have now:

Theorem 1. If the equations

$$
L^{(0)} \xi^{(0)}=\eta
$$

have a solution, then all the vectors $\xi^{(m)}$ are determined by $\xi^{(0)}$ and by the relations $\xi^{(1)}=0$ and the recurrence relations

$$
\xi^{(r)}=-S^{(2)} \xi^{(r-2)}-S^{(3)} \xi^{(r-3)}-\cdots-S^{(r)} \xi^{(0)} .
$$

In the particular case where

$$
\eta=\eta^{(0)}=(2 / 3,2 / 5,2 / i, \cdots),
$$

we have

$$
\xi^{(0)}=(\delta / \pi, 0,0,0, \cdots),
$$

and at most the first $r+1$ components of $\xi^{(r)}$ are different from zero. $\xi^{(0)}, \cdots, \xi^{(r)}$ are the solutions of the original system (3.6), if we use the first $r+1$ equations for determining the first $r+1$ unknowns and thereby neglect all terms inrolving the higher pouers of $\beta$ from the $r$-th power onwards. $\xi^{(0)}, \cdots, \xi^{(r)}$ also determine the cxact values of the first $r+1$ coefficients of the expansion of $T^{*}$ in pourers of $\beta$. 
The proof of theorem 1 follows immediately from lemma 1 and in particular from the fact that the $S^{(2 \nu)}, S^{(2 q+3)}$ involve many vanishing elements. The uniqueness of the $\xi^{(r)}$, and the existence of the $x_{n}$ (at least for sufficiently small values of $\beta$ ) will be proved in section 5 .

4. Limiting cases for the matrix $L$. Let

$$
P(t)=\Gamma(t+3 / 2) / \Gamma(t+1), \quad Q(t)=\Gamma(t+5 / 2) / \Gamma(t+1) .
$$

Then Theorem 1 states that the equations

$$
\sum_{m=0}^{\infty} l_{n, m}(\beta) x_{m}=h_{n} \quad(n=0,1,2, \cdots)
$$

can be solved by formal (i.e. not necessarily convergent) power series in $\beta$ if the equations

$$
4 L^{(0)} \xi \equiv\left\{\pi^{1 / 2} P(n) \sum_{m=1)}^{\infty} x_{m} P(m) / Q(n+m)\right\}=\left\{4 h_{n}\right\}
$$

have a solution $x_{m}=x_{m}^{(0)}$. We shall investigate (4.3) together with the limiting case $\beta \rightarrow \infty$. Levine and Schwinger [1] have shown that then (4.2) tends towards the system of linear equations

$$
L^{(\infty)} \xi \equiv\left\{\sum_{m=0}^{\infty} x_{m} /(n+m+2)\right\}=\mu\left\{h_{n}\right\}, \quad(n=0,1,2, \cdots)
$$

where $\mu$ is a constant.

We have to define first the linear space of admissible solutions $x_{m}$ from the nature of the problem. Since (3.1) is supposed to define the field in the aperture, and since the field cannot have a singularity in the center of the aperture, we must assume that

$$
\lim _{\epsilon \rightarrow 0} \sum_{m=0} x_{m}(1-\epsilon)^{m}
$$

exists. Since the original system (3.6) was set up merely in order to define the transmission coefficient, we shall assume that

$$
\sum_{m=0}^{\infty} x_{m} /(m+3 / 2)
$$

converges. This implies, that

$$
\sum_{m=0}^{\infty} x_{m} z^{m}
$$

converges for $|z|<1$ and therefore that the $x_{m}$ actually define the field in the aperture. Then we prove first:

Lemma 3. If the vector $\xi$ with the components $x_{m}$ satisfies (4.5) and (4.6), then the operators $L^{(0)}$ and $L^{(\infty)}$ are defined for $\xi$ in the sense that the sums in (4.3), (4.4) converge for $n=0,1,2, \cdots$

Proof: Let $Q(t)$ be defined as in (4.1) and let

$$
\tau_{m}=Q(m) / Q(n+m), \quad \sigma_{m}=\sum_{r=0}^{m} x_{r} /(r+3 / 2) .
$$

Then the partial sums of the series in (4.3) are

$$
\sum_{r=0}^{m} \tau_{r} x_{r} /(r+3 / 2)=\sum_{r=0}^{m-1}\left(\tau_{r}-\tau_{r+1}\right) \sigma_{r}+\tau_{m} \sigma_{m}
$$


where

$$
2 \tau_{r+1}-2 \tau_{r}=3 n P(r+1) /\{Q(n+r)[n+r+5 / 2]\} .
$$

Since the $\left|\sigma_{n}\right|$ are bounded and $\sum_{r}\left|\tau_{r}-\tau_{r+1}\right|$ converges, the sums in (4.3) also converge. The proof for the convergence of the sums in (4.4) is even simpler.

Theorem 2. If the equations $L^{(0)} \xi=\left\{h_{n}\right\}$ or $L^{(\infty)} \xi=\left\{h_{n}^{*}\right\}$ have a solution $\xi=\left\{x_{m}^{(0)}\right\}$ or $\xi=\left\{x_{m}^{(\infty)}\right\}$ satisfying (4.5) and (4.6), then the integral equations

$$
\begin{gathered}
\int_{0}^{1} f(v)(1-v)^{1 / 2}(1-v z)^{-1} d v=4 \pi^{-1 / 2} \sum_{n=0}^{\infty} z^{n} h_{n} n ! /(3 / 2)_{n}, \\
\int_{0}^{1} f^{*}(v) v(1-v z)^{-1} d v=\sum_{n=0}^{\infty} h_{n}^{*} z^{n},
\end{gathered}
$$

have analytic solutions

$$
f(v)=\sum_{m=0}^{\infty} v^{m} x_{m}^{(0)} \Gamma(m+3 / 2) / m !, \quad f^{*}(v)=\sum_{m=0}^{\infty} x_{m}^{(\infty)} v^{m} .
$$

The solutions are unique and they also solve the problems of moments

$$
\int_{0}^{1} f(v)(1-v)^{1 / 2} v^{n} d v=4 \pi^{-1 / 2} h_{n} n ! /(3 / 2)_{n}, \quad \int_{0}^{1} f^{*}(v) v^{n+1} d v=h_{n}^{*} .
$$

The integrals in (4.11) (4.12) are defined by

$$
\int_{0}^{1}=\lim _{\epsilon \rightarrow+0} \int_{0}^{1-c}
$$

Since a formal expansion of the left hand sides of (4.11) and (4.12) leads to the linear equations $L^{(0)} \xi=\left\{h_{n}\right\}$ and $L^{(\infty)} \xi=\left\{h_{n}^{*}\right\}$, it has only to be shown that, under the assumptions made about the $x_{m}$, such an expansion is legitimate. It suffices to prove that

$$
\lim _{\ell \rightarrow 0} \int_{0}^{1-e} f(v)(1-v)^{1 / 2} v^{n} d v=8 \pi^{-1} h_{n} n ! / \Gamma(n+3 / 2)
$$

where now $f(v)$ is defined by (4.13) and $h_{n}$ by $L^{(0)} \xi=\left\{h_{n}\right\}$. Since it follows from the assumption (4.5) about the $x_{m}$ that $f(v)$ converges absolutely and uniformly for $0 \leqq$ $v \leqq 1-\epsilon$, we may integrate term by term in (4.16). Putting $Y_{m}=x_{m}^{(0)} \Gamma(m+3 / 2) / m$ ! this gives (with $v=(1-\epsilon) W$ )

$$
\begin{aligned}
& \sum_{m=0}^{\infty} Y_{m} \int_{0}^{1-\epsilon} v^{n+m}(1-v)^{1 / 2} d v \\
& =\sum_{m=0}^{\infty} Y_{m}(1-\epsilon)^{n+m+1} \int_{0}^{1} W^{n+m}[1-(1-\epsilon) W]^{1 / 2} d W \\
& =\sum_{m=0}^{\infty} Y_{m}(1-\epsilon)^{n+m+1}(n+m+1)^{-1} F(-1 / 2, n+m+1, n+m+2 ; 1-\epsilon) \\
& =\sum_{m=0}^{\infty} Y_{m}(1-\epsilon)^{n+m+1}(n+m+1)^{-1} F(-1 / 2, n+m+1 ; n+m+2 ; 1) \\
& \quad \quad \quad \quad \sum_{m=0}^{\infty} Y_{m}(1-\epsilon)^{n+m+1}(n+m+1)^{-1}\{F(\cdots ; 1-\epsilon)-F(\cdots ; 1)\}
\end{aligned}
$$


According to Gauss's formula (cf. Whittaker-Watson [3])

$F(-1 / 2, n+m+1 ; n+m+2 ; 1)=(n+m+1) ! \Gamma(3 / 2) / \Gamma(n+m+5 / 2)$,

and from Abel's lemma and from lemma 3 it follows that

$\lim _{\epsilon \rightarrow 0} \sum_{n_{1}=0}^{\infty} Y_{m}(1-\epsilon)^{n+m+1} \Gamma(3 / 2)(n+m) ! /\left(\Gamma(n+m+5 / 2)=4 \pi^{-1 / 2} h_{n} n ! /(3 / 2)_{n}\right.$.

Now we have to show that the second sum in (4.19) tends towards zero as $\epsilon \rightarrow 0$. Because of (4.5) it suffices to show that

$c_{m, n}(\epsilon)$

$=\Gamma(m+3 / 2)[m !]^{-1}[n+m+1]^{-1}\{F(-1 / 2, n+m+1 ; n+m+2 ; 1-\epsilon)$

$-F(-1 / 2, n+m+1 ; n+m+2 ; 1)\}$

$=\Gamma(m+3 / 2)(2 m !)^{-1} \sum_{k=1}^{\infty}\left[1-(1-\epsilon)^{k+1}\right]$

$$
\cdot(1 / 2)_{k} /\{(k+1) !(n+m+k+2)\} \rightarrow 0
$$

as $\epsilon \rightarrow 0$ uniformly in $n, m$. We can prove that $\left|c_{m, n}(\epsilon)\right|<\epsilon$ by observing that $1-(1-\epsilon)^{k+1} \leqq(k+1) \epsilon$. This and (4.23) gives

$$
\begin{aligned}
\left|c_{m, n}(\epsilon)\right| & \leqq \epsilon \Gamma(m+3 / 2)(2 m !)^{-1} \sum_{k=0}^{\infty}(1 / 2)_{k}(n+m+k+2)^{-1}\{k !\}^{-1} \\
= & \epsilon \Gamma(m+3 / 2)\{2 m !(n+m+2)\}^{-1} F(1 / 2, n+m+2 ; n+m+3 ; 1) \\
= & \epsilon \Gamma(1 / 2) \Gamma(m+3 / 2)(n+m+1) !\{2 m ! \Gamma(n+m+5 / 2)\}^{-1} \\
= & \epsilon \frac{\pi^{1 / 2}}{2} \frac{(m+1)(m+2) \cdots(m+n+1)}{(m+3 / 2)(m+5 / 2) \cdots(m+n+3 / 2)} \leqq \epsilon \pi^{1 / 2} / 2<\epsilon .
\end{aligned}
$$

The uniqueness of the solution follows from

Lemma 4: If $\sum_{m=0}^{\infty} x_{m} /(m+3 / 2)$ converges, then for $0 \leqq v<1,(1-v)^{3 / 2}|f(v)|$ is bounded. The proof follows from summation by parts with the notation (4.8) and from the remark that

$$
\sum_{m=0}^{\infty} \Gamma(m+5 / 2)\left|\sigma_{m}\right| v^{m} /(m+1) ! \leqq C\left[(1-v)^{-3 / 2}-1\right] v^{-1},
$$

where $c$ does not depend on $v$.

Now we can show that (4.3) cannot have a null solution. Because then the difference $\phi(v)$ of two solutions of (4.11) would satisfy

$$
\int_{0}^{1} \phi(v)(1-v)^{1 / 2} v^{n} d v=0, \quad n=0,1,2, \cdots
$$

and therefore:

$$
\int_{0}^{1} \phi(v)(1-v)^{1 / 2}(1-v) v^{n} d v=0, \quad n=0,1,2, \cdots
$$

But $\phi(v)(1-v)^{3 / 2}$ would be a function continuous in $0 \leqq v \leqq 1$ according to lemma 4 and therefore $(4.27)$ shows that $\phi(v)(1-v)^{3 / 2}$ would be identically zero. 
Conclusions from theorem 1. The equivalence of the equations $L^{(0)} \xi=\left\{h_{m}\right\}$ and $L^{(\infty)} \xi=\left\{h_{m}^{*}\right\}$ to a problem of moments shows that these sets of linear equations are unstable in the following sense: Not unly may these equations have no solution at all, but this is certain to happen if we start with a set $\left\{h_{m}\right\}$ of right hand sides for which a solution exists and then change a finite number of the $h_{m}$ by an amount however small. In this case there does not even exist a continuous function $f(v)$ which satisfies (4.11) or (4.12) with the modified right hand sides.

The integral operators in (4.11), (4.12) are extensions of the linear operators defined by $L^{(0)}$ or $L^{(\infty)}$, since (4.11) or (4.12) may have a continuous solution $f(v)$ which is not analytic. Consequently, a quantity like the transmission coefficient

$$
T^{*}=\int_{0}^{1} f(v) v^{1 / 2} d v=\sum_{m=0}^{\infty} x_{m} /(m+3 / 2)
$$

can be defined even in cases where the $x_{m}$ do not exist. An easy example is offered by the equations

$$
\sum_{m=0}^{\infty} x_{m} /(n+m+2)=\mu /(n+3 / 2), \quad(n=0,1,2, \cdots)
$$

which were also investigated by Levine and Schwinger. The corresponding integral equation is

$$
\int_{0}^{1} f(v) v(1-v W)^{-1} d v=\mu \sum_{n=0}^{\infty} W^{n} /(n+3 / 2)=\mu \int_{0}^{1} v^{1 / 2} /(1-v W)^{-1} d v
$$

which gives

$$
f(v)=\mu v^{-1 / 2}, \quad I^{*}=\mu .
$$

In this case no set of $x_{m}$ satisfying (4.29) can exist. However, it is possible to find sequences of constants $Y_{m}^{(r)}$ such that

$$
\sum_{m=0}^{\infty} Y_{m}^{(r)}(m+n+2)^{-1}=\psi_{n}^{(r)}
$$

exist and

$$
\lim _{r \rightarrow \infty} \sum_{n=0}^{\infty}\left\{\psi_{n}^{(r)}-\mu /(n+3 / 2)\right\}^{2}=0, \quad \lim _{r \rightarrow \infty} \sum_{m=0}^{\infty} Y_{m}^{(r)} /(m+3 / 2)=\mu .
$$

For this purpose, we can choose the $Y_{m}^{(r)}$ from

$$
\sum_{m=0}^{\infty} Y_{m}^{(r)} v^{m}=\sum_{k=0}^{r}(1-v)^{k}(1 / 2)_{k} / k !
$$

The right hand side in (4.34) is a polynomial which approximates $v^{-1 / 2}$, since it is the $(r+1)$-th partial sum of $[1-(1-v)]^{-1 / 2}$. Clearly, the $Y_{m}^{(r)} \rightarrow \infty$ as $r \rightarrow \infty$.

5. Uniqueness and existence of the solution. Once a vector $\xi^{(0)}$ has been determined such that $L^{(0)} \xi^{(0)}=\eta$, where $\eta$ is the vector of the right hand sides in the original equations $L \xi=\eta$, we can determine $\xi$ from

$$
M \xi=\xi^{(0)}
$$


where, for all values of $\beta, M$ is defined by

$$
M=\mathfrak{g}+\sum_{p=1}^{\infty} \beta^{2 p} S^{(2 p)}+\sum_{q=0}^{\infty} \beta^{2 q+3} S^{(2 q+3)}
$$

Here $g$ denotes the identity. We shall call a vector $\xi$ bounded if $\sum\left|\xi_{m}\right|^{2}<\infty$ and we shall call a matrix $M$ bounded if there exists a constant $U>0$ such that for all bounded vectors $\xi$ :

$$
\xi^{*} M^{*} M \xi \leqq U^{2} \sum\left|\xi_{m}\right|^{2}
$$

where $M^{\prime}$ is the transposed matrix of $M$ and an asterisk denotes the conjugate complex quantity. $U$ is called an upper bound for $M$. It is well known that, if $U_{r}$ is an upper bound for $S^{(r)}(r=1,2,3 \cdots)$, the matrix $M$ in (5.2) has a bounded inverse $M^{-1}$ if

$$
\sum_{r=2}^{\infty} \beta^{r} U_{r}<1
$$

$M^{-1}$ can be obtained from a Neumann series. We can use this in order to prove:

Theorem 3. Let $L, M, \eta^{(0)}, \xi^{(0)}$ be defined by (3.5), (5.1), (3.27), (3.28). Then $M^{-1}$ exists and is bounded for sufficiently small values of $|\beta|<\beta_{0}$ and the equations $L \xi=\eta^{(0)}$ have exactly one solution $\xi$ which satisfies (4.5) and (4.6), namely $\xi=M^{-1} \xi^{(0)}$.

Proof: Let $V^{(r)}$ be matrices such that

$$
\left\{g+\sum_{r=2}^{\infty} \beta^{r} S^{(r)}\right\} g\left(\sum_{r=0}^{\infty} \beta^{r} V^{(r)}\right\}=\mathscr{J} .
$$

It is easily seen that the $V^{(r)}$ can be obtained from the $S^{(r)}$ by recurrence formulas. Let $U^{(r)}$ be upper bounds for the $S^{(r)}$ and assume that there exist constants $\Omega_{r}$ such that

$$
\left(1-\sum_{r=2}^{\infty} \beta^{r} U_{r}\right)\left(1+\sum_{r=2}^{\infty} \beta^{r} \Omega_{r}\right)=1 .
$$

This is true if

$$
1-\sum_{r=2}^{\infty} \beta^{r} U_{r}
$$

is convergent and positive for $0 \leqq \beta<\beta_{0}$. Then it can be shown that $\Omega_{r}$ is an upper bound for $V^{(r)}$. Since it can also be shown that $x_{m}$ (the $m$-th"component of $\xi=M^{-1} \xi^{(0)}$ ) is equal to the $m$-th component of

$$
\left\{\sum_{r=m}^{\infty} \beta^{r} V^{(r)}\right\} \xi_{0}
$$

it follows that

$$
\left|x_{m}\right| \leqq \sum_{r=m}^{\infty} \beta^{r} \Omega_{r}
$$

From this it can easily be shown that for $|\beta|<\beta_{0}$ condition (4.5) for the $x_{m}$ is satisfied. This proves the existence of $M^{-1}$ and of a bounded $\xi$ satisfying (4.5), (condition (4.6) 
is always satisfied for bounded $\xi$ ) if we can find $U_{r}$ which are sufficiently small. We have

Lemma 4. The matrices

$$
\left\{S^{(2)}\right\}^{t}, \quad R^{(t)}, \quad S^{(2 t+2)}, \quad S^{(2 q+3)}
$$

have as upper bounds

$$
\begin{gathered}
\pi\left(\pi^{2}-8\right)^{1 / 2} / 4, \quad 2^{1 / 2}\left(\pi^{2}-8\right)^{1 / 2} / t !, \quad\left(2 \pi^{2}-16\right)^{1 / 2} 2^{t+2}(1 / 2)_{t} /(t+1) ! \\
2^{a+1}\left(2 \pi^{2}-16\right)^{1 / 2} /(q+1) !
\end{gathered}
$$

The proof is elementary but laborious and will be omitted since the upper bounds are not the best possible ones.

In order to prove the uniqueness of the solution $\xi=M^{-1} \xi^{(0)}$ we observe first that $(M-g) \xi$ is bounded for every $\xi$ merely satisfying (4.5); provided that $\beta$ is so small

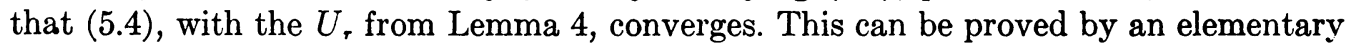
investigation of the $S^{(r)}$. Now if there is a $\xi^{*}$ satisfying (4.5) and (4.6) such that $L \xi^{*}=0$, we would have $M \xi^{*}=\xi^{*}+\zeta$ where $\zeta$ is bounded and $L^{(0)} \xi^{*}+L^{(0)} \zeta=0$. Now it follows from the equivalence of the operator $L^{(0)}$ to the operator of a moment problem (cf. Theorem 2) that $\xi^{*}+\zeta=0$. Therefore $\xi^{*}$ is bounded, and since $M^{-1}$ is bounded, $\xi^{*}$ must be zero since $M \xi^{*}=\xi^{*}+\zeta=0$.

No numerical values for the permissible ranges of $\beta$ are given since it is entirely possible that the inverse $M^{-1}$ exists for all values of $\beta$. This seems to be indicated by a result of Sommerfeld and Perron [5] who showed that for the related problem of the freely vibrating disc the real part of a resulting set of linear equations can be solved explicitly and without restrictions.

\section{REFERENCES}

[1] H. Levine and J. Schwinger, On the theory of diffraction by an aperture in an infinite plane screen. Phys. Review 74, No. 8, October (1948), 958-974.

[2] C. J. Bouwkamp, Theoretische en numerieke behandelung van de buigung door een ronde opening. Dissertation. Groningen (1941), 1-64.

[3] E. T. Whittaker and G. N. Watson, A course on modern analysis, Cambridge (1927).

[4] W. N. Bailey, Generalized hypergeometric series, Cambridge Tracts No. 32, London (1935).

[5] A. Sommerfeld, Die freischwingende Kolbenmembran. Annalen der Physik (5) 42, 389-420 (1943) and an addition in 6-th series, 2, 85-86 (1947). 